\title{
Abnormal Sexual Behavior During Sleep in Temporal Lobe Epilepsy: a Case Report
}

\author{
Zerrin Pelin ${ }^{1,2}$, Ece Yazla ${ }^{3}$ \\ ${ }^{1}$ Department of Neurology, Vocational School for Health-Related Professionals, Gazikent University, Gaziantep, Turkey \\ 2Department of Neurology, Somnus Sleep Disorders Center, Istanbul, Turkey \\ ${ }^{3}$ Department of Psychiatry, Erenköy Psychiatry and Neurology Training and Education Hospital, İstanbul, Turkey
}

\begin{abstract}
Herein, we describe a case who presented with abnormal sexual behaviour during sleep. Video-electroencephalography monitoring during sleep revealed an abnormality suggesting an epileptic basis. The patient was successfully treated with carbamazepin. The psychiatric symptoms that were thought to be related to abnormal sexual behaviours were controlled with antipsychotic treatment. Our findings strongly emphasize the fact that efforts should be spent to increase awareness of seizure activity at night, which can be misinterpreted as benign parasomnias. Such a misinterpretation may have serious consequences, such as insufficient seizure control, progressive personality changes, and cognitive impairment.
\end{abstract}

Key Words: Sexual behaviour during sleep, nocturnal seizure, temporal lobe epilepsy, sexsomnia

Received: 28.07.2011 Accepted: 17.09.2011

\section{Introduction}

In clinical practice, the differential diagnosis of attacks occurring during sleep should be made by neurologists, particularly epileptologists. The range of possible diagnoses includes sleep disorders, psychiatric disturbances, and epileptic seizures (1). These attacks may follow different behavioural patterns including bizarre motor behaviour, autonomic activation or abnormal sexual behaviour. Sexual behaviour during sleep includes masturbation, sexual fondling, anal and oral sex, as well as heterosexual and homosexual intercourse, all typically presenting in peculiar or bizarre circumstances; i.e. covers almost all aspects of human sexual behaviour (2). There is an expanding set of sleep disorders and other nocturnal disorders associated with abnormal sexual behaviours or the misperception of sexual behaviours (3).

Herein, we report a patient masturbating during sleep. Diagnosis was established after a detailed clinical and neurophysiologic examination.

\section{Case Report}

A 31-year-old man was admitted to the Outpatient Clinic of the Department of Neurology with complaints of muscle contractions, meaningless talk, taking off his clothes, and atypical sexual behaviours during sleep. His past medical history revealed no neurological or psychiatric disorders before the onset of the complaints. His medical history started eight years previously with laughing, banging on the wall and clapping his hands during sleep. These behaviours occurred three to four times a night and at any time during the night. Some of these episodes were characterized with masturbation, unintelligible talk, moving around, and visual and auditory hallucinations that lasted approximately 15 minutes. During the episodes, he was not able to recognize anybody, even his sons, and it was almost impossible to stop him. Sometimes these behaviours occurred during daytime napping. He imitated animal sounds, such as the meow of a cat or the bray of a donkey. His body was contracted but he did not turn black and blue or have incontinence.

Following the onset of the complaints, behavioural changes as well as personality changes occurred. There was a marked increase in his libido. He was convinced that all women were in love with him so he asked to have sex with almost all of the women around him, without caring who they were. He lost almost all of his friends because of his inappropriate and aggressive behaviours. He was unable to work and earn money. He was referred to a psychiatrist two years after the onset of the symptoms for the first time, and treatment was started with ziprasidone $60 \mathrm{mg}$ per day. Because no improvement had been noted in his symptoms despite four months of treatment with ziprasidone, he was referred to another psychiatrist who established the diagnosis of psychomotor epilepsy. The patient was started on valproic acid and topiramate polytherapy, and further dose adjustments were made in small increments. The patient was on irregular treatment for four years, including some periods without any medication. He started to lose consciousness for about 15 seconds whenever he felt excited. He did not turn black and blue or have urinary incontinence during these periods. His symptoms were refractory to treat- 
ment. He had a convulsion just once when he neglected his treatment. He turned purple and remained unconscious with foamy salivation for about one hour.

Video-electroencephalography (VEEG) monitoring and polysomnographic recordings during sleep were planned when the patient was admitted to the Outpatient Clinic of the Department of Neurology in our hospital. Nocturnal VEEG revealed an episode of masturbation during sleep. During that particular episode, hypersynchronous delta waves with a frequency of 2-3 $\mathrm{Hz}$ together with muscle artefacts that were more prominent on the anterior temporal and temporal regions were observed (Figure 1). There was no evidence of pathological signs indicating epileptic activity during episodes of masturbation. During the masturbation episode, the EEG revealed neuronal hyperexcitability in the right temporal area (Figure 2). Cranial magnetic resonance imaging (MRI) showed that the Sylvian fissures were enlarged, and the cortical sulci were deeper than normal bilaterally. The patient underwent a battery of neurocognitive tests that revealed impaired attention and memory. Psychiatric evaluation with the dissociative experiences scale (DES) eliminated the possibility of a dissociative state. His DES score was found to be 7.8. Valproic acid therapy was discontinued after appropriate dose adjustments made in small decrements. The patient was started on carbamazepine. Topiramate was continued in the same dose ( $250 \mathrm{mg}$ daily), and the dose of carbamazepine was increased to $1200 \mathrm{mg}$ daily. His complaints occurring during sleep completely disappeared with this treatment; however, he started being suspicious and jealous of his wife. He was reported to become very aggressive even losing control, and harming his wife and children. Risperidone $4 \mathrm{mg}$ daily) was added to the treatment regimen for the treatment of new symptoms. His suspicious and aggressive behaviours were improved with risperidone therapy; however, the patient started to complain about a decline in sexual functions. His prolactin level was $40.3 \mathrm{nmol} / \mathrm{L}$ (normal reference range, 3.46-19.4 $\mathrm{nmol} / \mathrm{L})$. He was also complaining about feeling unhappy and exhausted. Extrapyramidal signs and symptoms were consid-

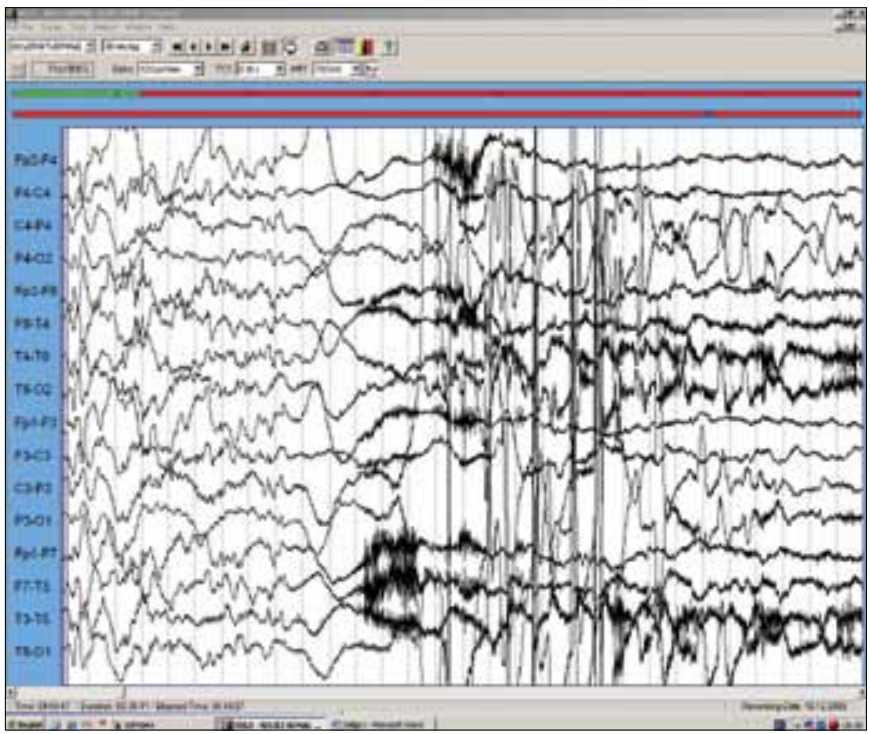

Figure 1. Nocturnal EEG shows hypersyncronous delta waves during the episode of masturbation ered to be complications of risperidone therapy. Due to the complications of the therapy, risperidone was changed to aripiprazole therapy (10 $\mathrm{mg}$ daily). The dose of carbamazepine was increased to $1500 \mathrm{mg}$ daily, while the dose of topiramate was reduced to $175 \mathrm{mg}$ daily. On his last control visit, it was noted that his complaints had disappeared with treatment, and there was no evidence of an active pathologic process. The patient was scheduled for regular follow-up.

\section{Discussion}

The difficulties in distinguishing nocturnal epileptic seizures from parasomnias reflect just one aspect of the intriguing issue of the physiopathological relationships between all types of paroxysmal motor behaviours during sleep. In fact, the aetiopathogenesis of nocturnal phenomena, even events of different aetiology, i.e. epileptic or non-epileptic in nature that can coexist in the same subject, is a matter of debate (1).

In complex partial seizure, it is possible to misinterpret an abnormal sexual behavior during sleep as a parasomnia episode. Such a misinterpretation may lead to insufficient seizure control, misdiagnosis, and progressive cognitive impairment (4). Herein, we describe a case presenting with masturbation episodes, walking around and non-sense talking during sleep, like a "somnambulism plus" events. Previously, two cases (one male, one female) were reported by Guilleminault et al. where there were abrupt and violent masturbation episodes recurring during the night. In that report, one patient had no previous history of epilepsy and it was shown that there was a right mesiofrontal focus activity on the EEG and good response to the antiepileptic treatment (5). Seizures arising in the right temporal lobe are more likely to cause orgasm or sexual automatisms (6). The occurrence of the behaviours at any time during the night, multiple occurrences of the episodes, the short duration of the episodes, and the excellent response of the patient to carbamazepine therapy supported the epileptic basis in our patient.

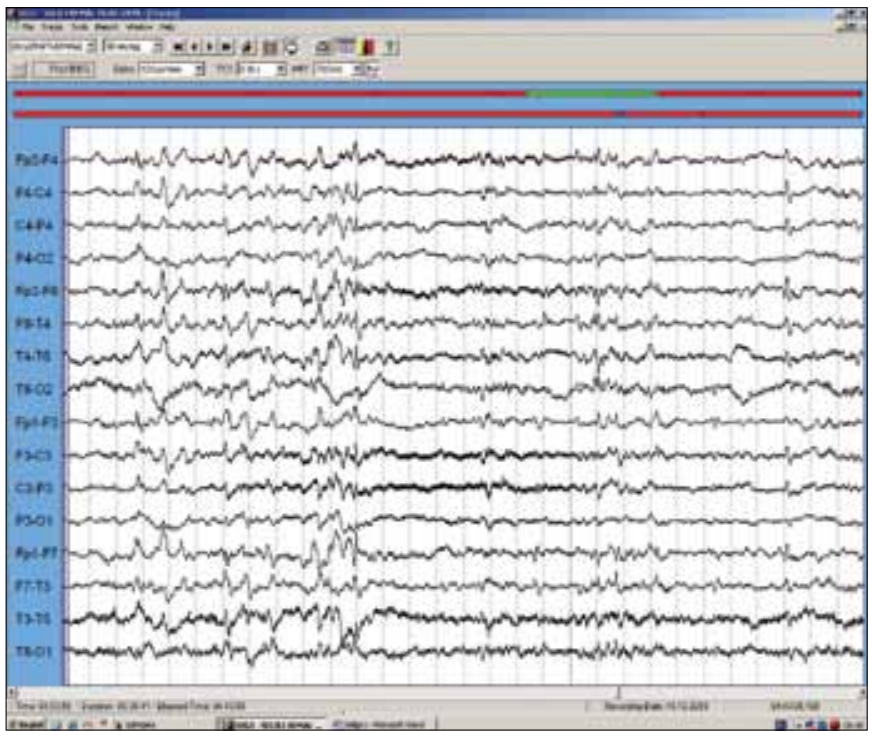

Figure 2. Right temporal neuronal hyperexcitability in periods outside the masturbation episode 
Various clinical features of sexsomnia, including sexual vocalization and talking, masturbation, sexual fondling, and sexual intercourse have been described. The great majority of the cases of sexsomnia in the literature are males (7). The differential diagnosis of parasomnia is established by nocturnal EEG and polysomnography (PSG) recordings with video monitoring. These techniques enable neurologists to eliminate the possible diagnosis of nocturnal epileptic seizures, especially nocturnal frontal or temporal lobe seizures. Since sexual hyperarousal, ictal orgasm, and ictal sexual automatisms are also seen in sleep-related seizures, it is difficult to distinguish sexsomnia from sleep-related seizures. In our case, the possible diagnosis of sexsomnia was not clearly ruled out by nocturnal EEG with video monitoring. Hypersynchronous delta waves, as in our case, were not considered as a specific EEG findings for the events associated with parasomnia, although some reports presented a burst of hypersynchronous delta waves appearing just before the abnormal behaviour and persisting in the initial part of the events (8).

Chronic change in personality is very common in temporal lobe epilepsy. Behavioural changes typically follow the onset of seizures. These changes may continue for years and gradually become more intense (9). The dramatic personality change with increased viscosity, anger, and sexuality was clearly observed in our case. His past medical history revealed no psychiatric disorders, thus the appearance of psychotic symptoms following the cessation of nocturnal seizures using carbamazepine might have resulted from a forced normalization.

Frontal and temporal lobe epilepsies comprise the largest group of pure sleep epilepsies. Recent evidence indicates that secondary generalization during sleep is less prominent in frontal than temporal lobe epilepsies (1). Several authors have shown that sleep organization is more severely disrupted in patients with temporal lobe epilepsy than patients with extratemporal lobe epilepsy. Sleep improvement is likely to play a role in the therapeutic effects of antiepileptic drugs. Among all conventional antiepileptic drugs, both acute and chronic administration of carbamazepine provides the most effective stabilization of sleep patterns in healthy controls, and in epileptic patients. Touchon et al. (10) showed that, in patients with temporal lobe epilepsy, sleep disturbance was improved with carbamazepine therapy. The sleep-stabilizing effect of carbamazepine might have led to improvement in this patient.

\section{Conclusion}

Distinguishing nocturnal seizures from parasomnia is often difficult and sometimes impossible on clinical grounds alone. Efforts should be made to increase awareness of seizure activity at night by using V-EEG, polysomnography and response to the medical treatment.

\section{Conflict of Interest}

No conflict of interest was declared by the authors.

\section{References}

1. Tinuper P, Provini F, Bisulli F, Vignatelli L, Plazzi G, Vetrugno R, et al. Movement disorders in sleep: guidelines for differentiating epileptic from non-epileptic motor phenomena arising from sleep. Sleep Med Rev 2007;11:255-67. [CrossRef]

2. Trajanovic NN, Mangan M, Shapiro CM. Sexual behaviour in sleep: an internet survey. Soc Psychiatry Psychiatr Epidemiol 2007;42:1024-31. [CrossRef]

3. Schenck $\mathrm{CH}$, Arnulf I, Mahowald MW. Sleep and sex: what can go wrong? A review of the literature on sleep related disorders and abnormal sexual behaviors and experiences. Sleep 2007;30:683-702.

4. Silvestri R, Bromfield E. Recurrent nightmares and disorders of arousal in temporal lobe epilepsy. Brain Res Bull 2004;63:369-76. [CrossRef]

5. Guilleminault C, Moscovitch A, Yuen K, Poyares D. Atypical sexual behaviour during sleep. Psychosomatic Med 2002;64:328-36.

6. Arnedo V, Parker-Menzer K, Devinsky O. Forced spousal intercourse after seizures. Epilepsy Behav 2009;16:563-4. [CrossRef]

7. Béjot $Y$, Juenet N, Garrouty R, Maltaverne D, Nicolleau L, Giroud $M$, et al. Sexsomnia: an uncommon variety of parasomnia. Clin Neurol Neurosurg 2010;112:72-5. [CrossRef]

8. Andersen ML, Poyares D, Alves RS, Skomro R, Tufik S. Sexomnia: Abnormal sexual behaviour during sleep. Brain Res Rev 2007;56:271-82. [CrossRef]

9. Devinsky J, Schachter S. Norman Geschwind's contribution to the understanding of behavioral changes in temporal lobe epilepsy: the February 1974 lecture. Epilepsy Behav 2009;15:41724. [CrossRef]

10. Touchon J, Baldy-Moulinier M, Billiard M, Besset A, Cadilhac J. Sleep organization and epilepsy. Epilepsy Res Suppl 1991;2:73-81. 